\section{Atypical Ductal Hyperplasia: Factors predicting upstaging to carcinoma}

\author{
Kristen Elstner ${ }^{1 *}$, Sinem Gultekin ${ }^{1}$, Wendy Vincent ${ }^{2}$ and Sanjay \\ Warrier ${ }^{1.2}$
}

${ }^{1}$ The University of Sydney, Faculty of Medicine, Sydney, NSW, Australia

${ }^{2}$ Royal Prince Alfred Hospital, Missenden Road, Camperdown, NSW, Australia

\section{Abstract}

Aim: Percutaneous core needle biopsy (CNB) is considered the gold standard technique for initial histological diagnosis of suspicious breast lesions seen on screening mammogram, but it is less reliable for diagnosing atypical ductal hyperplasia (ADH) due to significant rates of diagnosis upstaging to malignant disease after excision biopsy. The purpose of this study was to identify factors that predict diagnosis upstage to carcinoma in patients diagnosed with ADH on core biopsy.

Methods: A retrospective database search identified 52 consecutive CNB of suspicious breast lesions revealing pure ADH. Inclusion criteria included asymptomatic women presenting for screening mammogram, who subsequently underwent surgical excision. Logistic regression analysis evaluated clinical, radiological, and histological factors.

Results: A total of 52 patients with ADH on CNB were identified who met our criteria. Twentysix of 52 patients $(50 \%)$ were upstaged to ductal carcinoma in situ or invasive carcinoma, based on histological interpretation of the surgically excised specimen. Lesion size was showed to be a statistically significant predictor on univariable logistic regression analysis. Multivariate logistic regression analysis revealed Asian ethnicity and lesion size as independent predictors of malignancy ( $p=0.050$ and 0.011 , respectively). Conversely, women of Middle Eastern and European origin and lesions $<15 \mathrm{~mm}$ on mammography were negative predictors of malignancy.

Conclusion: Lesion size $\geq 15 \mathrm{~mm}$ on mammography and Asian ethnicity are independent risk factors for breast carcinoma in asymptomatic patients diagnosed with ADH on CNB.

\section{More Information}

*Address for Correspondence: Dr. Kristen Elstner, The University of Sydney, Faculty of Medicine, Sydney, NSW, Australia,

Tel: (+61) 0400310 289;

Email: kristenelstner@hotmail.com

Submitted: 10 June 2020

Approved: 03 July2020

Published: 06 July 2020

How to cite this article: Elstner K, Gultekin S, Vincent W, Warrier S. Atypical Ductal Hyperplasia: Factors predicting upstaging to carcinoma. Arch Surg Clin Res. 2020; 4: 011017.

DOI: 10.29328/journal.ascr.1001045

ORCiD: orcid.org/0000-0002-2224-7165

Copyright: @ 2020 Elstner K, et al. This is an open access article distributed under the Creative Commons Attribution License, which permits unrestricted use, distribution, and reproduction in any medium, provided the original work is properly cited.

(W) Check for updates

OPEN ACCESS

\section{Introduction}

Atypical Ductal Hyperplasia (ADH) is a common histopathological diagnosis in the current era of populationbased mammographic screening. With improved diagnostic imaging, and increasing awareness and popularity of breast screening programmes, a growing number of asymptomatic women are presenting for routine mammographic screening. Following this trend is an increasing detection rate of subclinical or non-palpable breast lesions which appear as suspicious on diagnostic imaging [1,2]. These radiologic abnormalities are recommended for further investigation, commonly performed using core needle biopsy (CNB). This offers a histological diagnosis explaining the mammographic findings and is crucial for determining further management.

Up to $15 \%$ of CNB of suspicious breast lesions seen on screening mammogram yield a diagnosis of Atypical Ductal Hyperplasia (ADH) [3]. Considered a potential early precursor of invasive breast cancer, $\mathrm{ADH}$ is a high-risk lesion which confers a four- to fivefold risk of subsequent development of invasive malignancy, as compared to the general population [4-6]. ADH most frequently presents as clustered (micro) calcification on mammography. It is variably defined histologically as (1) a lesion with some, but not all, of the features of low grade Ductal Carcinoma in Situ (DCIS), or (2) a lesion with all the cytologic and architectural features of low grade DCIS but involving no more than one duct or measuring less than $2 \mathrm{~mm}$ in diameter [2,5,7-9]. Because ADH may co-exist with DCIS and its distinction from DCIS is partly quantitative, it is sometimes not possible to distinguish between these 2 lesions in the limited samples provided by CNB. Additionally, the limited sampling offered by core biopsy has the potential to miss a co-existing invasive cancer [2]. For these reasons, in many regions of the world the preferred treatment of biopsy-proven ADH of the breast is surgical excision, to fully appreciate the true extent and nature of disease [10].

Lesions initially diagnosed as ADH on core biopsy, but 
which are found to harbour DCIS or invasive carcinoma on surgical excision are termed 'upstaged' lesions. The rate of ADH upstaging after CNB reported in the literature varies between $11.5-62 \%$ [8]. The maximal upstaging rate that is generally considered acceptable is $2 \%$; this is the risk of malignancy that is widely accepted to define lesions as 'probably benign' and for which short-term imaging followup is appropriate as an alternative to surgical excision [1113].

Several authors have previously attempted to identify risk factors associated with $\mathrm{ADH}$ upstaging to carcinoma, to define a low-risk population in whom surgery might be avoided [1,3,8,11,12,14-27]. However, to date, no individual or combined criteria have been consistently able to predict a subset of the population who might be at low risk of upstaging [8,14-16,23,26,28,29]. There is an abundance of conflicting evidence in the literature on this subject. While some authors have previously discussed options of surgery versus short term follow-up, many continue to recommend a mandatory excisional biopsy due to the unacceptably high risk of diagnosis upstage to more advanced lesions [20].

The primary objectives of this study were to determine the ADH upstaging rate to carcinoma at our institutions, and to elicit identifiable factors predictive of breast malignancy in asymptomatic patients diagnosed with ADH on CNB. Our secondary objective was to identify a subset of patients who are at low risk of harbouring carcinoma and who might therefore safely avoid surgery.

\section{Methods}

This study was approved by the local Human Research Ethics Review Committee. In this analysis, patients with $\mathrm{ADH}$ on core biopsy were retrospectively identified from the prospectively maintained BreastScreen NSW (Cancer Institute) database.

Between July 1, 2008 and December 31, 2014, 66 patients were identified from the BreastScreen database whose CNB pathology demonstrated $\mathrm{ADH}$. Inclusion criteria consisted of female gender, asymptomatic presentation, and CNB demonstrating pure ADH. Patients were excluded from this analysis if core biopsy revealed a coexisting malignant lesion (ductal carcinoma in situ or invasive malignancy) $(n=7)$. Other exclusion criteria included patients who did not proceed to surgical excision $(n=3)$, medical records unavailable for review $(n=4)$, or a palpable mass associated with the mammographic abnormality $(n=0)$. This resulted in a study population of 52 patients (Figure 1).

The preferred method of biopsy for mammographic lesions presenting as asymmetry, distortion or density was ultrasound guided 14-gauge core biopsy needles, or stereotactic guidance if the lesion was not visible under ultrasound. Where the primary lesion involved

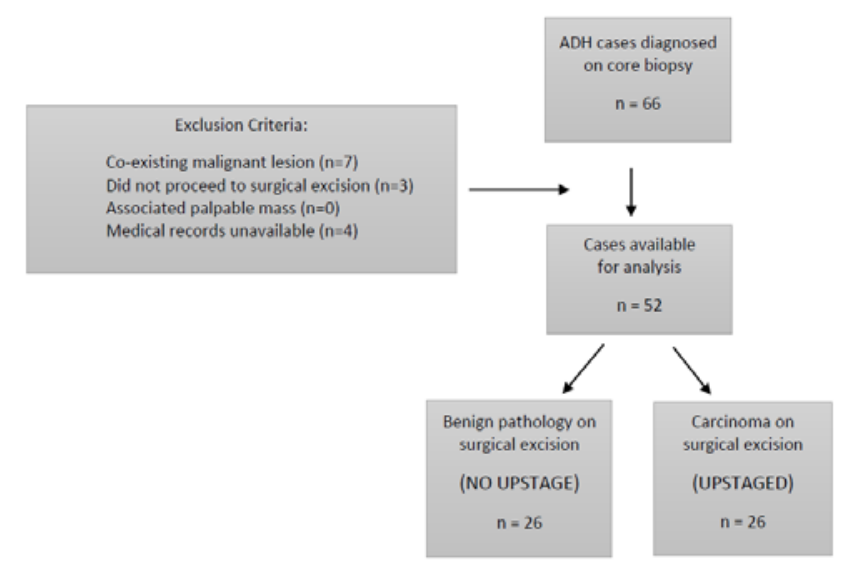

Figure 1

microcalcifications, biopsies were performed under stereotactic guidance using 10-gauge vacuum-assisted core biopsy needle.

For the purpose of this study, a single specialized breast radiologist re-evaluated all mammograms. Amount of calcification in millimetres on the original screening mammogram was recorded, as was complete or incomplete removal of calcifications after core biopsy. Mammographic breast density was scaled in percentiles as " $<25 \%$ glandular" if the breast was almost entirely fat, "25\% - 50\% glandular" if there were scattered fibroglandular densities, " $51 \%-75 \%$ glandular" if the breast tissue was heterogeneously dense, and "> 75\% glandular" if the breast tissue was extremely dense.

Patients diagnosed with ADH on CNB were further grouped into 'carcinoma' or 'benign', based on the final histological diagnosis of the excised surgical specimen. Histopathologic diagnoses were retrieved from original pathology reports of surgical excision specimens. Biopsy and surgical specimens were interpreted using standard criteria by pathologists specializing in breast pathology. The 'carcinoma' (or 'upstaged') group consisted of a final diagnosis of ductal carcinoma in situ (DCIS) and/or invasive ductal carcinoma (IDC). The 'benign' group included ductal hyperplasia without atypia, atypical ductal hyperplasia, atypical lobular hyperplasia, lobular carcinoma in situ, and other proliferative diseases.

Descriptive statistics were used to present characteristics of the study population with XLSTAT, using student t-test to compare continuous variables across patient groups. For univariable and multivariable analyses, logistic regression modelling was performed using the statistical package R. Each factor was first tested individually for association with histological upstaging using a univariable logistic regression model. Variables with $p$ value $<0.1$ were then considered as candidates for the multivariable logistic regression model. Final $p$ values of $\leq 0.05$ were considered as statistically significant. 


\section{Results}

Between July 1, 2008 and December 31, 2014, 52 asymptomatic patients were identified with $\mathrm{ADH}$ at core biopsy performed for suspicious lesions on screening mammogram, who subsequently underwent surgical excision. The study included women who ranged in age from 42 to 83 years (mean 56 years in both groups). Of the 52 patients, 26 (50\%) were upstaged to carcinoma on surgical excision (DCIS $n=17$, IDC $n=9$ ) (Table 1). Mean lesion size in the benign group was $14.2 \mathrm{~mm}$, and $22.7 \mathrm{~mm}$ in the carcinoma group, although this difference was not statistically significant.

Table 2 summarizes the upstaging rates and distribution in all patients based on clinical, radiologic, and histologic characteristics. $88.5 \%(n=46)$ of patients were over the age of 50 at the time of ADH diagnosis. There was no significant difference in the risk of upstaging between the 2 groups with respect to use of Hormone Replacement Therapy or family history of breast cancer (first- or second- degree relatives). None of the women included in this analysis had a personal history of breast cancer. There was no statistically significant difference between the upstage rates for biopsies performed using 10-gauge vacuum-assisted stereotactic $(n=15 / 35$; $43 \%$ ), and 14-gauge ultrasound biopsy ( $n=11 / 17 ; 65 \%)$ modalities.

'Ethnicity' referred to patient's country of origin. 28 patients identified as Australian born and constituted the majority of patients included in this study. 9 patients identified as Asian and included patients from south and south-eastern Asia (Vietnam, Philippines, Laos and Bangladesh). 7 patients identified as Middle Eastern in origin (Lebanon, Israel, and Iraq). 8 patients were included in the 'Other' category and included 1 patient from Africa, with the remaining 7 patients originating from continental Europe.

Histological analysis of the core biopsies demonstrated focal ADH in 61.5\% $(n=32)$ and multifocal ADH in 38.5\% $(n=20)$ of patients; there was no difference in the upstage rates $(50 \%)$ between those with focal versus multifocal ADH $(p=1.000)$. Complete removal of calcifications at time of

\begin{tabular}{|c|c|c|}
\hline Table 1: Characteristics of benign and carcinoma groups. & $\begin{array}{c}\text { Benign Group } \\
\text { (no upstage) }\end{array}$ & $\begin{array}{c}\text { Carcinoma Group } \\
\text { (yes upstage) }\end{array}$ \\
\hline Patient number & 26 & 26 DCIS 16 (61.5\%) \\
\hline Mean age (years) & 56.4 & IDC 10 (38.5\%) \\
\hline Mean lesion size (mm) & (range 42 - 68) & (range 43 - 83) \\
\hline & 14.2 & 22.7 \\
\hline Tumour grade & (range 2 - 65) & (range 4-65) \\
\hline & & Low: DCIS 9; IDC 8 \\
\hline & & Intermediate: DCIS 4; IDC 1 \\
\hline IDC: invasive ductal carcinoma; DCIS: ductal carcinoma in situ.
\end{tabular}

biopsy demonstrated an upstage risk of $27 \%(n=4 / 15)$, with incomplete removal demonstrating a 55\% upgrade risk $(n=$ $16 / 29)$, although this difference did not quite reach statistical significance ( $p=0.055)$.

Lesion size (on mammogram) $<15 \mathrm{~mm}$ or $\geq 15 \mathrm{~mm}$ was found to be the only variable associated with upstaging which was statistically significant on univariable logistic regression analysis ( $\mathrm{p}$ 0.02343).

Ethnicity and lesion size reached independent significance on multivariable analysis (p 0.050 and 0.011 , respectively). Lesions $\geq 15 \mathrm{~mm}$ demonstrated a 5 times higher odds of being upgraded to malignancy (odds ratio 5.064; p 0.011) compared with a lesion $<15 \mathrm{~mm}$. Women of Asian ethnicity exhibited the highest risk of being upstaged (odds ratio 3.2), whereas those originating from the Middle East and 'Other' category (primarily European) demonstrated a significantly less chance of being upstaged (odds ratio 0.27 and 0.20 , respectively) (Table 3 ).

A Receiver Operating Characteristic (ROC) curve was calculated using ethnicity and lesion size characteristics. The area under the ROC curve is estimated at 0.627 (a fair predictive model). Applying the results of the multivariable logistic regression and ROC model to our population, patients included in both 'Other' (primary European) ethnicity $(n=4)$ and Middle Eastern $(n=4)$ categories, with lesion size $<15 \mathrm{~mm}$ on mammography, demonstrated $0 \%$ upstaging rates.

\section{Discussion}

\section{Natural history of ADH}

Underpinning some of the controversy surrounding the management of ADH is the poor understanding of its natural history. One theory suggested by Page, et al. [5,30]. and Rosai [31]. is that ADH and DCIS constitute somewhat arbitrary points along a continuum of ductal neoplasias, each with an increasing degree of atypia and proliferation, which corresponds to an increasing relative risk of developing invasive breast carcinoma. In 1992, although Page and Rogers attempted to standardize pathologic diagnostic criteria for DCIS and ADH, they noted that there is no absolute division between 'cancer yes' and 'cancer no,' a viewpoint which imposes borderline diagnostic categories and challenges the traditional dichotomous mode of diagnosis [7].

Over the past 3 decades, although the existence of borderline lesions has been well described, their natural history has not. It remains unclear whether ADH represents a true precursor lesion or rather is a histologic manifestation of a tissue bed at increased risk of malignant transformation. If atypia represents a direct precursor to malignancy, one would expect a preponderance of subsequent breast cancers to occur in the ipsilateral breast, with shared histologic features and a shorter time to occurrence. By contrast, if 
Table 2: Clinical, radiological and pathology variables and their associated distribution between benign and carcinoma groups. $p$ values $<1.0$ used for multivariable logical regression analysis are bolded and underlined.

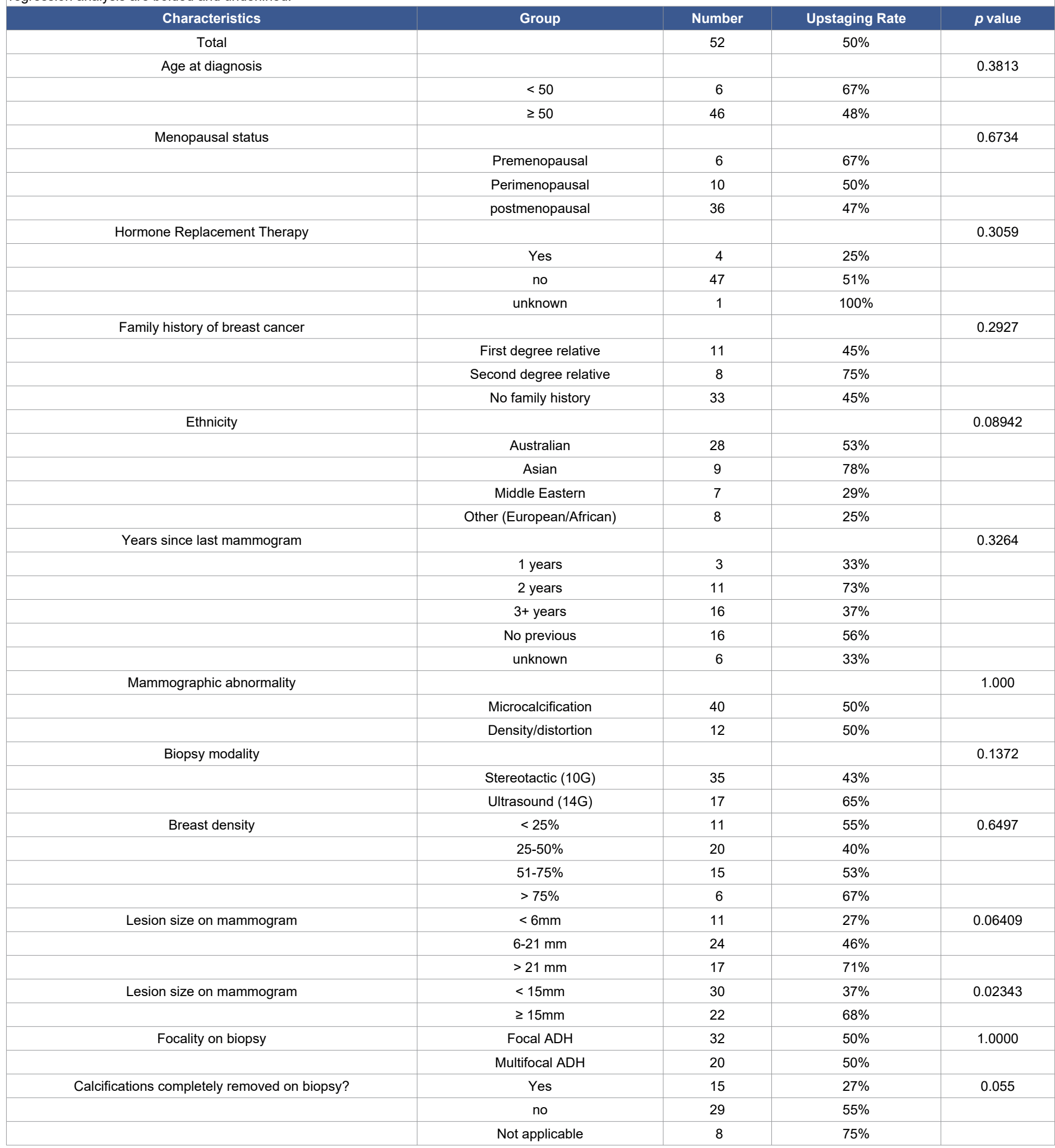

Table 3: Results of multivariable analysis demonstrating that ethnicity and lesion size are statistically significant variables when estimating risk of ADH upstaging to carcinoma after core needle biopsy.

\begin{tabular}{|c|c|c|c|}
\hline Multivariate characteristics & Odds Ratio & $95 \%$ Confidence Interval & 0.050 \\
\hline Asian ethnicity & 3.2 & $0.495-20.7$ & $0.037-2.02$ \\
\hline Middle East & 0.27 & $0.030-1.42$ & 0.011 \\
\hline Other (European) & 0.20 & $19.7-1.30$ & 0.12 \\
\hline Lesion size $(\geq 15 \mathrm{~mm})$ & 5.1 & \\
\hline
\end{tabular}


an atypia is a general risk indicator, any subsequent breast cancers would be expected to occur with equal frequency in both breasts, with variable histology and time to occurrence. In fact, there is evidence for both theories. In a study by Hartmann et al of 698 women with atypical hyperplasia followed for a mean of 12.5 years, 143 developed breast cancer. There was a 2:1 ratio of ipsilateral versus contralateral breast cancer. The ipsilateral predominance is marked in the first 5 years, lending credibility to the argument of a precursor phenotype. This study also demonstrated longer term elevated risk involving both breasts, giving strength to the general increased risk theory [32].

The question remains: can we preoperatively identify those population subsets at the far end of the continuum who are at high risk of harbouring invasive disease ('cancer yes'), and can we identify those at the other end of the spectrum, who are at low risk and might safely avoid surgery altogether ('cancer no')?

\section{Scoring systems}

Several groups have previously attempted to identify clinical, radiological, and pathological patterns to enable prediction of those who are at low risk of being upstaged from $\mathrm{ADH}$ to malignancy $[1,3,8,11,12,14-27]$. Their aim was to identify a subset of women who were considered low risk for upstaging and could possibly avoid surgery, and instead, undergo short term radiological follow-up and clinical review. While several factors have been found to be associated with risk of upstaging, no factor alone or in combination has been consistently associated with an acceptably low risk of diagnosis upstaging. The results of most studies show a risk of upstaging generally above the $2 \%$ threshold and surgical excision therefore remained the gold standard recommendation for all populations [8,14,16,23,26,28,29].

Ko, et al. [14] developed a prediction algorithm to determine the probability of upstaging of ADH to malignancy in ultrasound-guided CNB. Their study of 74 patients demonstrated that age $>50$ years, microcalcification on mammography, lesion size $>15 \mathrm{~mm}$, and a palpable lesion were independent predictors of malignancy. A scoring system was developed whereby the scores for each significant factor were added, resulting in a total score ranging between 0 and 14.5. Ko's algorithm demonstrated a negative predictive value of $100 \%$ for a score $\leq 3.5$. The algorithm was able to identify a subset of their study population $(n=6)$ whose diagnosis was likely to be benign. Ko's study included a validation dataset of 54 patients, where 15 patients were classified as 'probably benign' with scores $\leq 3.5$, and again demonstrated that no patients were upstaged to malignancy. In an external validation study of 107 patients, Bendifallah, et al. [28] assessed the statistical performance of the Ko algorithm in an independent multicentre population, and achieved a negative predictive value of $89 \%$. They concluded that this scoring algorithm demonstrated a lack of reproducibility and was not sufficiently accurate to define a low risk subset of the population who could safely avoid surgery.

Forgeard, et al. [20] in a retrospective series of 116 patients, demonstrated that lesion size and extent of ADH were significant predictors of malignancy. From these results, they defined a group of patients with lesion size < $6 \mathrm{~mm}$ and complete removal of calcifications on biopsy who demonstrated a 0\% risk of upstaging. Caplain, et al. [29] working from the same institution as Forgeard, prospectively assessed Forgeard's guidelines using a series of 124 patients and a median follow-up of 30 months (range 5-55 months). Conformity rate was $92 \%$. Patients with lesion size $<6 \mathrm{~mm}$ and complete removal of calcifications were assigned to undergo clinical and radiological follow-up only. Of the 61 patients who did not undergo surgery, one patient developed a malignancy in the contralateral breast after 2 years, and a second patient developed a malignancy after 3 years in a different quadrant of the ipsilateral breast. Although acknowledging that longer follow-up analysis is required to increase the precision of their results, Caplain's conclusion was that these guidelines do allow a subset of patients to be safely followed-up with yearly mammograms and examination rather than surgery. However, when Forgeard's algorithm is applied to the current study's population, a different conclusion is reached. 7 patients were identified with lesion size $<6 \mathrm{~mm}$ and complete removal of calcification on biopsy, with 4 out of 7 patients demonstrating carcinoma on surgical excision (upstage rate of 57\%). A low risk population who might safely avoid surgery has not been demonstrated with Forgeard's algorithm is applied to this study population.

\section{Selected other factors of interest}

To the best of our knowledge, this is the first study to demonstrate that ethnicity may play a role in the risk of ADH upgrade to carcinoma. The data from this study does demonstrate statistical significance. However, this must be interpreted with caution given the significant heterogeneity of the broad ethnic categories in this analysis, and the relatively small patient numbers included within each category. The authors regard these findings as a point of interest to be studied further and not a definite established link.

Numerous other factors have been previously found to be statistically significant on evaluation of malignancy risk. Larger lesion size $[12,18,33]$, multifocal ADH $[15,16]$, older age [23] and micropapillary pattern on histology $[15,26]$. have been associated with higher risk of malignancy. Conversely, unifocal ADH $[19,26]$, complete removal of calcifications $[11,15,19,27,34]$, have been variably associated with a lower risk of ADH upstage to malignancy. Nonetheless, data analysis from many of these studies failed to reach the $2 \%$ threshold 
and therefore continued to recommend surgical excision as the primary management strategy.

The concept of ADH with 'marked' or 'severe' cytologic atypia is an evolving area of interest. The new entity described by Allison, et al. [35] as "ADH suspicious for DCIS" is a qualitative feature which may be more discerning in predicting upstage than many of the quantitative factors described above. Similarly, Adrales, et al. described 'marked atypia' as a predictor of malignancy, but the histologic criteria was not precisely defined, making replication difficult $[29,36]$. Nguyen, et al. [3] also noted 'significant atypia' as a specific risk factor for upstaging and recommended surgical excision. Whether the addition of another arbitrary category along the continuum of atypia will serve to be of use is something that will remain to be seen. Future studies will help discern whether this demonstrates added prognostic benefit. However, uniformity of its definition and terminology will need to be established before it can become a useful value.

Limitations of this study include its retrospective nature and small size. This limited our data and meant not all factors of interest could be examined. In the current study, 3 patients did not proceed to surgical excision and were therefore excluded. It is possible that cases with a lower probability of malignancy were recommended for imaging follow-up rather than surgical excision, and may have affected our results. As mentioned previously, our findings of ethnicity as a significant risk factor must be interpreted with caution, due to the necessity of categorizing large geographic areas and ethnic diversity.

\section{Conclusion}

At our institution, the rate of ADH upstaging to carcinoma after core biopsy was clinically significant at $50 \%$. The present study demonstrates that lesion size $\geq 15 \mathrm{~mm}$ is an independent predictor of malignancy, and that Asian ethnicity may also be an independent predictor. To the best of our knowledge, this is the only study to date indicating that ethnicity may play a significant role in the risk of ADH upstaging to carcinoma. Additionally, our data suggest that asymptomatic women with a diagnosis of ADH after core needle biopsy who present with lesions $\leq 15 \mathrm{~mm}$, and who are of European or Middle Eastern origin, could potentially be managed safely with short term imaging follow-up as opposed to surgical excision. However, given the considerable heterogeneity of results and conclusions from other studies, it is suggested that specialists continue to manage their patients on a case-by-case basis, with multidisciplinary input, until clear evidence-based recommendations can be made from large, prospectively assessed studies. Further research is warranted into the possibility of ethnicity as a risk factor for diagnosis upstaging.

\section{Acknowledgement}

Louise Helby and Peterlou Statistics for statistical analysis.
There are no sources of funding to disclose.

All authors (KE, SG, WV, SW) declare that there are no conflicts of interest.

\section{References}

1. Ancona A, Capodieci M, Galiano A, Mangieri F, Lorusso V, et al. Vacuum-assisted biopsy diagnosis of atypical ductal hyperplasia and patient management. Radiol Med. 2011; 116: 276-291.

PubMed: https://www.ncbi.nlm.nih.gov/pubmed/21225358

2. Masood S, Rosa M. Borderline breast lesions: diagnostic challenges and clinical implications. Adv Anat Pathol. 2011; 18: 190-198. PubMed: https://www.ncbi.nlm.nih.gov/pubmed/21490436

3. Nguyen CV, Albarracin CT, Whitman GJ, Lopez A, Sneige N, et al. Atypical ductal hyperplasia in directional vacuum-assisted biopsy of breast microcalcifications: considerations for surgical excision. Ann of Surg Oncol. 2011; 18: 752-761.

PubMed: https://www.ncbi.nlm.nih.gov/pubmed/20972636

4. Dupont WD, Parl FF, Hartmann WH, Brinton LA, Winfield AC, et al. Breast cancer risk associated with proliferative breast disease and atypical hyperplasia. Cancer. 1993; 71: 1258-1265.

PubMed: https://www.ncbi.nlm.nih.gov/pubmed/8435803

5. Page DL, Dupont WD, Rogers LW, Rados MS. Atypical hyperplastic lesions of the female breast. A long-term follow-up study. Cancer. 1985; 55: 2698-2708.

PubMed: https://pubmed.ncbi.nlm.nih.gov/2986821/

6. Dupont WD, Page DL. Risk factors for breast cancer in women with proliferative breast cancer. N Engl J Med. 1985; 312: 146-151. PubMed: https://pubmed.ncbi.nlm.nih.gov/3965932/

7. Page DL, Lowell WR. Combined histologic and cytologic criteria for the diagnosis of mammary and atypical ductal hyperplasia. Hum Pathol. 1992; 23: 1095-1097.

PubMed: https://www.ncbi.nlm.nih.gov/pubmed/1328030

8. McGhan LJ, Pockaj BA, Wasif N, Giurescu ME, McCullough AE, et al. Atypical ductal hyperplasia on core biopsy: an automatic trigger for excisional biopsy? Ann Surg Oncol. 2012; 19: 3264-3269.

PubMed: https://pubmed.ncbi.nlm.nih.gov/22878619/

9. VandenBussche CJ, Khouri N, Sbaity E, Tsangaris TN, Vang R, et al. Borderline atypical ductal hyperplasia/low-grade ductal carcinoma in situ on breast needle core biopsy should be managed conservatively. Am J Surg Pathol. 2013; 37: 913-923.

PubMed: https://www.ncbi.nlm.nih.gov/pubmed/23598968

10. Sinn HP, Elsawaf Z, Helmchen B, Aulmann S. Early breast cancer precursor lesions: lessons learned from molecular and clinical studies. Breast Care. 2010; 5: 218-226.

PubMed: https://www.ncbi.nlm.nih.gov/pmc/articles/PMC3346166/

11. Villa A, Tagliafico A, Chiesa F, Chiaramondia M, Friedman D, et al. Atypical ductal hyperplasia diagnosed at 11-gauge vacuum-assisted breast biopsy performed on suspicious clustered microcalcifications: could patients without residual microcalcification be managed conservatively? AJR. 2011; 197: 1012-1018.

PubMed: https://www.ncbi.nlm.nih.gov/pubmed/21940593

12. Ko E, Han W, Lee JW, Cho J, Kim EK, et al. Scoring system for predicting malignancy in patients diagnosed with atypical ductal hyperplasia at ultrasound-guided core needle biopsy. Breast Cancer Res Treat 2008; 112: 189-195.

PubMed: https://www.ncbi.nlm.nih.gov/pubmed/18060577

13. American College of Radiology. Breast imaging reporting and data system (BI-RADS). $4^{\text {th }}$ ed. Reston, VA: American College of Radiology; 2003. 
14. Polat AK, Kanbour-Shakir A, Andacoglu O, Polat AV, Johnson R, et al. Atypical hyperplasia on core biopsy: is further surgery needed? Am J Med Sci. 2012; 344: 28-31.

PubMed: https://www.ncbi.nlm.nih.gov/pubmed/22205116

15. Wagoner MJ, Laronga C, Acs G. Extent and histologic pattern of atypical ductal hyperplasia present on core needle biopsy specimens of the breast can predict ductal carcinoma in situ in subsequent excision. Am J Clin Pathol. 2009; 131: 112-21.

PubMed: https://www.ncbi.nlm.nih.gov/pubmed/19095574

16. Deshaies I, Provencher L, Jacob S, et al. Factors associated with upgrading to malignancy at surgery of atypical ductal hyperplasia diagnosed on core biopsy. Breast 2011; 20: 50-55.

PubMed: https://www.ncbi.nlm.nih.gov/pubmed/20619647

17. Dominici L, Liao GS, Brock J. Large needle core biopsy of atypical ductal hyperplasia: results of surgical excision. Breast J. 2012; 18: 506-508. PubMed: https://www.ncbi.nlm.nih.gov/pubmed/22897750

18. Hong ZJ, Chu CH, Fan, HL, Hsu HM, Chen CJ, et al. Factors predictive of breast cancer in open biopsy in cases with atypical ductal hyperplasia diagnosed by ultrasound-guided core needle biopsy. Eur J Surg Oncol. 2011; 37: 758-764.

PubMed: https://www.ncbi.nlm.nih.gov/pubmed/21764539

19. Sneige N, Lim SC, Whitman GJ, Krishnamurthy S, Sahin AA, et al. Atypical ductal hyperplasia diagnosis by directional vacuum-assisted stereotactic biopsy of breast microcalcification. Am J Clin Pathol. 2003; 119: 248-253.

PubMed: https://www.ncbi.nlm.nih.gov/pubmed/12579995

20. Forgeard C, Benchaib M, Guerin N, Thiesse P, Mignotte H, et al. Is surgical biopsy mandatory in case of atypical ductal hyperplasia on 11 -gauge core needle biopsy? A retrospective study of 300 patients. Am J Surg. 2008; 196: 339-345.

PubMed: https://www.ncbi.nlm.nih.gov/pubmed/18585676

21. Mesurolle B, Perez JC, Azzumea F, et al. Atypical ductal hyperplasia diagnosed at sonographically guided core needle biopsy: frequency, final surgical outcome, and factors associated with underestimation. AJR 2013; 202: 1389-94

PubMed: https://www.ncbi.nlm.nih.gov/pubmed/24848840/

22. Menes TS, Rosenberg R, Balch S, Jaffer S, Kerlikowske $\mathrm{K}$, et al. Upgrade of high-risk breast lesions detected on mammography in the breast cancer surveillance consortium. Am J Surg. 2014; 207: 24-31. PubMed:: https://pubmed.ncbi.nlm.nih.gov/24112677

23. Chae BJ, Lee A, Song BJ, Jung SS. Predictive factors for breast cancer in patients diagnosed atypical ductal hyperplasia at core needle biopsy. World J Surg Oncol. 2009; 7: 77.

PubMed: https://www.ncbi.nlm.nih.gov/pmc/articles/PMC2771003/

24. Catteau X, Simon P, Noël JC. Predictors of invasive breast cancer in mammographically detected microcalcification in patients with a core biopsy diagnosis of flat epithelial atypia, atypical ductal hyperplasia or ductal carcinoma in situ and recommendations for a selective approach to sentinel node biopsy. Pathol Res Pract. 2012; 208: 217220.

PubMed: https://pubmed.ncbi.nlm.nih.gov/22445178/

25. Gümüş $H$, Mills $P$, Gumus $M$, Fish $D$, Jones $S$, et al. Factors that impact the upgrading of atypical ductal hyperplasia. Diagn Interv Radiol. 2013; 19: 91-96.

PubMed: https://www.ncbi.nlm.nih.gov/pubmed/23019055

26. Ely KA, Carter BA, Jensen RA, Simpson JF, Page DL. Core biopsy of the breast with atypical ductal hyperplasia. A probabilistic approach to reporting. Am J Surg Pathol. 2001; 25: 1017-1021.

PubMed: https://pubmed.ncbi.nlm.nih.gov/11474285/

27. Kohr JR, Eby PR, Allison KH, DeMartini WB, Gutierrez RL, et al. Risk of upgrade of atypical ductal hyperplasia after stereotactic breast biopsy: effects of number of foci and complete removal of calcifications. Radiology 2010; 255: 723-730.

PubMed: https://www.ncbi.nlm.nih.gov/pubmed/20173103

28. Bendifallah S, Defert S, Chabbert-Buffet N, Maurin N, Chopier J, et al. Scoring to predict the possibility of upgrades to malignancy in atypical ductal hyperplasia diagnosed by an 11-gauge vacuum-assisted biopsy device: an external validation study. Eur J Cancer. 012; 48: 30-36. PubMed: https://www.ncbi.nlm.nih.gov/pubmed/22100905

29. Caplain, A, Drouet $Y$, Peyron M, Peix M, Faure C, et al. Management of patients diagnosed with atypical ductal hyperplasia by vacuumassisted core biopsy: a prospective assessment of the guidelines used at our institution. Am J Surg. 014; 208: 260-267. PubMed: https://www.ncbi.nlm.nih.gov/pubmed/24680949

30. Page DL. Cancer risk assessment in benign breast biopsies. Hum Pathol. 1986; 17: 871-874

PubMed: https://www.ncbi.nlm.nih.gov/pubmed/3759071

31. Rosai J. Borderline epithelial lesions of the breast. Am J Surg Pathol. 1991; 15: 209-221.

PubMed: https://pubmed.ncbi.nlm.nih.gov/1847606/

32. Hartmann LC, Radisky DC, Frost MH, Santen RJ, Vierkant RA, et al. Understanding the premalignant potential of atypical hyperplasia through its natural history: a longitudinal cohort study. Cancer Prev Res. 2014; 7: 211-217.

PubMed: https://www.ncbi.nlm.nih.gov/pmc/articles/PMC4167687/

33. Jackman RJ, Nowels KW, Shepard MJ, Finkelstein SI, Marzoni FA, Jr et al. Stereotaxic large core needle biopsy of 450 nonpalpable breast lesions with surgical correction in lesions with cancer or atypical hyperplasia. Radiology. 1994; 193: 91-5.

PubMed: https://www.ncbi.nlm.nih.gov/pubmed/8090927

34. Jackman RJ, Birdwell RL, Ikeda DM. Atypical ductal hyperplasia: can some lesions be defined as probably benign after stereotactic 11-gauge vacuum-assisted biopsy, eliminating the recommendation for surgical excision? Radiology. 2002; 224: 548-554. PubMed: https://www.ncbi.nlm.nih.gov/pubmed/12147855

35. Allison KH, Eby PR, Kohr J, DeMartini WB, Lehman CD, et al. Atypical ductal hyperplasia on vacuum-assisted breast biopsy: suspicion for ductal carcinoma in situ can stratify patients at high risk of upgrade. Hum Pathol. 2011; 42: 41-50.

PubMed: https://www.ncbi.nlm.nih.gov/pubmed/20970167

36. Adrales G, Turk P, Wallace T, Bird R, Norton HJ, et al. Is surgical excision necessary for atypical ductal hyperplasia of the breast diagnosed by Mammotome? Am J Surg. 2000; 180: 313-315. PubMed: https://pubmed.ncbi.nlm.nih.gov/11113443/ 